\title{
Star formation properties in barred galaxies
}

\author{
Zhi-Min Zhou ${ }^{1}$, Chen $\mathrm{Cao}^{2}$ and Hong $\mathrm{Wu}^{1}$ \\ ${ }^{1}$ National Astronomical Observatories, Chinese Academy of Sciences, Beijing 100012, China \\ ${ }^{2}$ School of Space Science and Physics, Shandong University at Weihai, Weihai, Shandong \\ 264209, China \\ email: zmzhou@bao.ac.cn
}

\begin{abstract}
Stellar bars are important structures for the internal secular evolution of galaxies. They can drive gas into the central region of galaxies, and result in an enhancement of star formation activity there. Previous studies are limited in the comparisons between barred and unbarred galaxies. Here we try to investigate the connection between star formation activities and different bars, based on multi-wavelength data in a sample of barred spirals. We find that there is no clearly trend of the surface star formation rates in different structures along the bar strength. In addition, there is larger scatter for the properties of star formation activity in the galaxies with middle-strength bars, which may indicate that a variety of star formation stages are more likely associated with these bars.
\end{abstract}

Keywords. galaxies: evolution — galaxies: photometry — galaxies: structure

\section{Introduction}

Stellar bars have been found to be common structures in disc galaxies, and they are believed to be the important internal drivers to secular evolution of galaxies (Kormendy \& Kennicutt 2004). Bars are able to make the angular momentum of gas and stars redistribution in galactic disks, include large-scale streaming motions in the gas and stars, and result in an increase in the gas mass and enhancement of star formation activity in the inner regions of galaxies (e.g., Ho et al. 1997, Athanassoula 2003).

There are strong evidences for the bar-driven secular evolution, both theoretical and numerical. Numerical simulations have established that the gravitational torque of the large-scale bar can make the gas lose angular momentum, and then drive it down to the galactic central regions (Athanassoula 1992, Sellwood \& Wilkinson 1993). Many observations also support that barred spirals have higher molecular gas concentrations and enhanced star formation activity in the central kiloparsec than in unbarred systems (e.g., Sakamoto et al. 1999, Sheth et al. 2005). However, the connection between bars and star formation activity is less clear, and previous studies are limited in the comparisons between barred and unbarred galaxies. To investigate their correlation, we use multiwavelength data from ultraviolet (UV) to infrared (IR), and try to analyzes a sample of nearby barred galaxies with both weak and strong bars, including from early to late Hubble types spirals.

\section{Sample and Data}

Our galaxy sample was drawn from three Spitzer local programs: the Spitzer Infrared Nearby Galaxies Survey (SINGS; Kennicutt et al. 2003), the Local Volume Legacy (LVL; Dale et al. 2009), and the Infrared Hubble Atlas (Pahre et al. 2004). From all nearby galaxies in these programs, we took galaxies with bars (Hubble type of SB and SAB), 


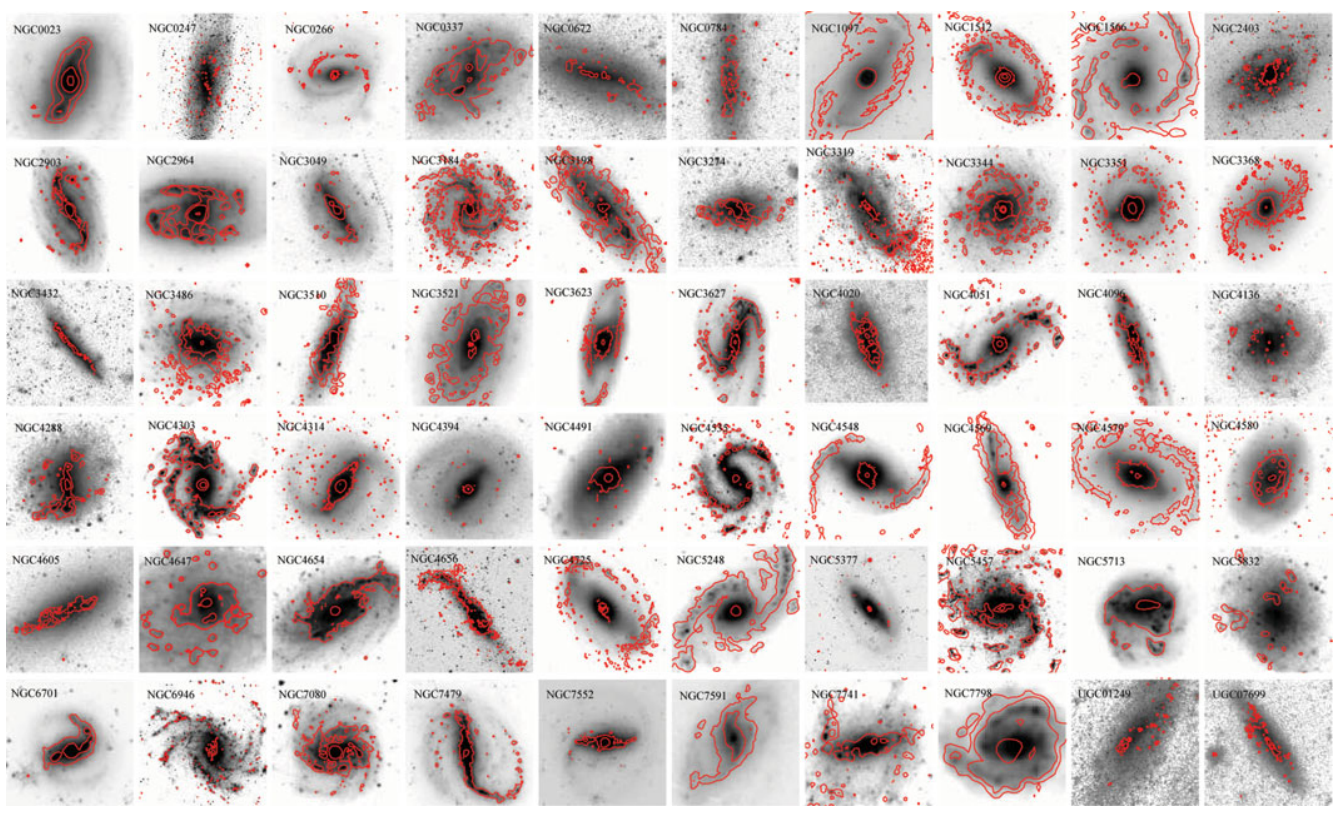

Figure 1. The mass images of the samples. Overplotted are the SFR imaging contours. The images are derived using our data and the corresponding conversion equations. The contours are in arbitrary units, optimized to show and identify the structures of the star formation complexes. The galaxy names are specified in the panels, and north is up and east to the left.

and removed apparently edge-on galaxies and objects with close neighbors. In the final sample, there are $34 \mathrm{SB}$ galaxies and $26 \mathrm{SAB}$ galaxies encompassing the Hubble types SaSm.

In order to investigate the properties of barred galaxies, we archived UV images observed by Galaxy Evolution Explorer (GALEX; Martin et al. 2005) and IR images from the Spitzer Space Telescope (Werner et al. 2004) data archive. In addition, the narrowband $\mathrm{H} \alpha$ images from ground-based telescopes were collected to analyze the star formation activities in our sample galaxies. Figure 1 show the images of our sample.

\section{Analysis and Results}

To further explore possible correlations between the star formation activity in different structural regions and the stellar bars, we performed image decomposition on the $3.6 \mu \mathrm{m}$ images, which can nicely trace the stellar compositions in nearby galaxies. Based on the decomposition results, we obtained the galaxy structural parameters, estimated the bar strength using ellipticity-based parameter $\mathrm{f}_{b a r}$, and derived the star formation activities in different structural regions, i.e., bulges, bars, disks, and the global galaxies.

In Figure 2, we compared the surface SFRs to the bar strength. In general, under visual inspection we see that there is no clearly trend of the surface SFRs in four panels along the bar strength, although there are weak trends such that the surface SFRs in bulges and global galaxies increase with the bar strength along with large scatter. In addition, there is no major discernible difference between early-type and late-type galaxies in their global, bar and disk regions. It is noteworthy that the bulges in our sample have larger scatter around at the middle strength of the bar, which may indicate that a variety of star formation stages in the central region are likely associated with the stellar bars with middle strength, not bars too weak, or too strong. In general, bar-driven secular 

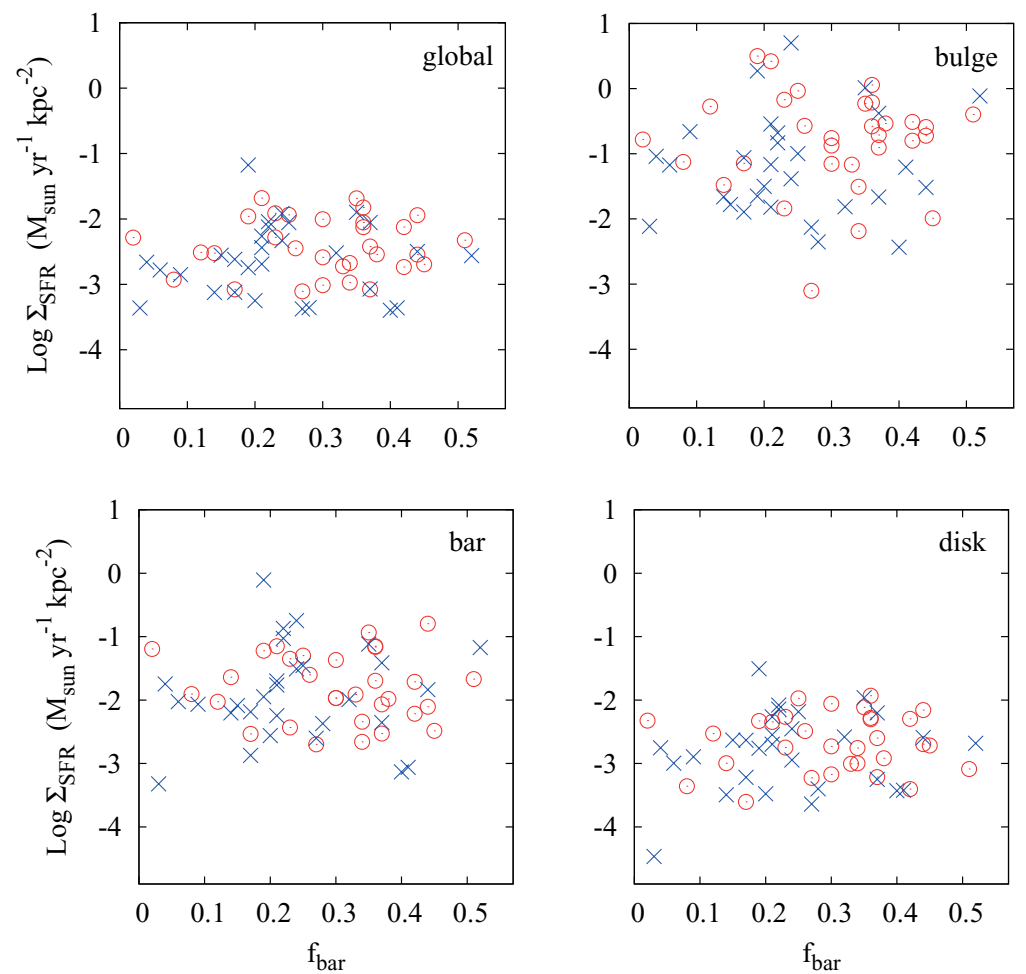

Figure 2. The surface star formation rates as a function of the bar strength $\mathrm{f}_{b a r}$. The surface star formation rates in the four panels are form different galactic structures, including bulges, bars and disks, as well as the global galaxies. The four panels use the same signals, circles for early-type galaxies $(1 \leqslant \mathrm{~T} \leqslant 4)$ and crosses for late-type ones $(5 \leqslant \mathrm{~T} \leqslant 9)$.

evolution is a complex process, and depends on many factors, such as time scales and the availability of gas (Coelho \& Gadotti 2011). Therefore, in the effect of bars, the star formation activity in the central region of galaxies is likely located in different evolutional stages, even with similar galactic bars.

\section{References}

Athanassoula, E. 2003, MNRAS, 341, 1179

Athanassoula, E. 1992, MNRAS, 259, 345

Coelho, P. \& Gadotti, D. A. 2011, arXiv1111.1736

Dale, D. A., et al. 2009, ApJ, 703, 517

Ho, L. C., Filippenko, A. V., \& Sargent, W. L. W. 1997, ApJ, 487, 591

Kennicutt, R. C. et al. 2003, PASP, 115, 928

Kormendy, J. \& Kennicutt, R. C. 2004, ARAA, 42, 603

Martin, D. C., et al. 2005, ApJ, 619, 1

Pahre, M. A., Ashby, M. L. N., Fazio, G. G., \& Willner, S. P. 2004, ApJS, 154, 235

Sakamoto, K., Okumura, S. K., Ishizuki, S., \& Scoville, N. Z. 1999, ApJ, 525, 691

Sellwood, J. A. \& Wilkinson, A. 1993, Rep. Prog. Phys., 56, 173

Sheth, K., Vogel, S. N., Regan, M. W., Thornley, M. D., \& Teuben, P. J. 2005, ApJ, 632, 217

Werner, M. W., et al. 2004, ApJS, 154, 1 